\title{
EULER'S RATIO-SUM FORMULA IN PROJECTIVE-METRIC SPACES
}

\author{
ÁRPÁD KURUSA AND JÓZSEF KOZMA
}

\begin{abstract}
We prove that Euler's ratio-sum formula is valid in a projectivemetric space if and only if it is either elliptic, hyperbolic, or Minkowskian.
\end{abstract}

\section{INTRODUCtion}

There has always been quite a broad interest (see $[10,4,5,12,13])$ in L. Euler's classical ratio-sum formula

$$
\frac{d(A, O)}{d(O, X)}+\frac{d(B, O)}{d(O, Y)}+\frac{d(C, O)}{d(O, Z)}+2=\frac{d(A, O)}{d(O, X)} \cdot \frac{d(B, O)}{d(O, Y)} \cdot \frac{d(C, O)}{d(O, Z)}
$$

for triangles $A B C \triangle$ in the Euclidean plane, where $O$ is any point outside of the sides (lines) of $A B C \triangle$, and $X=A O \cap B C, Y=B O \cap C A, Z=C O \cap A B$. In Euler's original paper [3] $O$ is an interior point of $A B C \triangle$, and we assume so in this paper too.

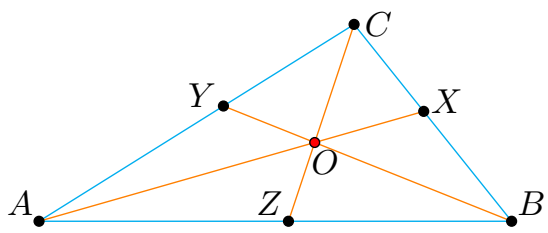

Already Euler started to look for analogous results on the sphere [3] (see also [9]), and by [10] we also have the hyperbolic analogue.

In this article, we give appropriate and unified generalizations of (1.1) to constant curvature planes in Theorem 2.2 with very simple proof.

Then we look for generalizations for projective-metric spaces, but eventually conclude in Theorem 4.5, that among the projective-metric spaces Euler's ratiosum formula characterizes the elliptic geometry, the Minkowskian geometries, and the hyperbolic geometry.

\section{NotATIONS AND PRELIMINARIES}

Points of $\mathbb{R}^{n}$ are denoted as $A, B, \ldots$, vectors are $\overrightarrow{A B}$ or $\boldsymbol{a}, \boldsymbol{b}, \ldots$ Latter notations are also used for points if the origin is fixed. Open segment with endpoints $A$ and $B$ is denoted by $\overline{A B}, \bar{A} B$ is the ray starting from $A$ passing through $B$, and the line through $A$ and $B$ is denoted by $A B$. The Euclidean scalar product is $\langle\cdot, \cdot\rangle$.

1991 Mathematics Subject Classification. 53A35; 51M09, 52A20.

Key words and phrases. Projective metrics, Hilbert geometry, Minkowski geometry, ellipses, triangle, tangential ratio.

This research was supported by NFSR of Hungary (NKFIH) under grant numbers K 116451 and KH 18129630. 
The affine ratio $(A, B ; C)$ of the collinear points $A, B$ and $C \neq B$ is defined by $(A, B ; C) \overrightarrow{B C}=\overrightarrow{A C}$. The affine cross ratio of the collinear points $A, B, C \neq B$, and $D \neq A$ is $(A, B ; C, D)=(A, B ; C) /(A, B ; D)$ [1, page 243].

Let $(\mathcal{M}, d)$ be a metric space given in a set $\mathcal{M}$ with the metric $d$. If $\mathcal{M}$ is a projective space $\mathbb{P}^{n}$ or an affine space $\mathbb{R}^{n} \subset \mathbb{P}^{n}$ or a proper open convex subset of $\mathbb{R}^{n}$ for some $n \in \mathbb{N}$, and the metric $d$ is complete, continuous with respect to the standard topology of $\mathbb{P}^{n}$, and the geodesic lines of $d$ are exactly the non-empty intersection of $\mathcal{M}$ with the straight lines, then the metric $d$ is called projective.

If $\mathcal{M}=\mathbb{P}^{n}$, and the geodesic lines of $d$ are isometric with a Euclidean circle; or $\mathcal{M} \subseteq \mathbb{R}^{n}$, and the geodesic lines of $d$ are isometric with a Euclidean straight line, then $(\mathcal{M}, d)$ is called a projective-metric space of dimension $n$ (see [1, p. 115] and [14, p. 188]). Such projective-metric spaces are called of elliptic, parabolic or hyperbolic type according to whether $\mathcal{M}$ is $\mathbb{P}^{n}, \mathbb{R}^{n}$, or a proper convex subset of $\mathbb{R}^{n}$. The projective-metric spaces of the latter two types are called straight [2, p. 1].

The geodesics of a projective-metric space of elliptic type have equal lengths, so we can set their length to $\pi$ by simply multiplying the projective metric with an appropriate positive constant. Therefore we assume from now on that

projective-metric spaces of elliptic type have geodesics of length $\pi$.

If $A, B$ are different points in $\mathcal{M}$, and $C \in(A B \cap \mathcal{M}) \backslash\{B\}$, then the real number

$$
\langle A, B ; C\rangle_{d}=\left\{\begin{aligned}
\frac{d(A, C)}{d(C, B)}, & \text { if } C \in \overline{A B}, \\
-\frac{d(A, C)}{d(C, B)}, & \text { otherwise }
\end{aligned}\right.
$$

is called the metric ratio of the triple $(A, B, C)$. In Minkowski geometries this coincides with the affine ratio.

The following theorem is proved in [8], but its partial proofs can be found in earlier publications too (see [3], [9] and [10]).

Theorem 2.1. In the Euclidean plane we have:

(1) If $O$ is a point in the open triangle $A B C \triangle$, and $X=A O \cap B C, Y=$ $B O \cap C A$, and $Z=C O \cap A B$, then (1.1) is valid, and the sum of any two of the numbers

$$
p=\frac{d(A, O) d(O, X)}{d(A, X)}, q=\frac{d(B, O) d(O, Y)}{d(B, Y)}, r=\frac{d(C, O) d(O, Z)}{d(C, Z)}
$$

is bigger than the third one.

(2) If three segments $\overline{A X}, \overline{B Y}$, and $\overline{C Z}$ has a common point $O$ such that (1.1) is fulfilled, and the sum of any two of the numbers in (2.2) is bigger than the third one, then the segments $\overline{A X}, \overline{B Y}$, and $\overline{C Z}$ can be turned around $O$ so that every one of $X, Y, Z$ lays on the side of the triangle $A B C \triangle$ opposite to the appropriate vertex $X, Y, Z$, respectively. 
The sequence of algebraically equivalent equations

$$
\begin{aligned}
\frac{1}{1+a}+\frac{1}{1+b}+\frac{1}{1+c} & =1 \\
(1+b)(1+c)+(1+a)(1+c)+(1+a)(1+b) & =(1+a)(1+b)(1+c) \\
a+b+c+2 & =a b c
\end{aligned}
$$

is crucial in the proof of Theorem 2.1, and implies that (1.1) is equivalent to

$$
\frac{d(O, X)}{d(A, X)}+\frac{d(O, Y)}{d(B, Y)}+\frac{d(O, Z)}{d(C, Z)}=1 .
$$

To find and prove the appropriate version of Euler's ratio-sum in constant curvature spaces, we use the projector map $\tilde{\mu}$ which projects a point given in polar coordinates $(\boldsymbol{u}, r)$ at a point $O$ in the constant curvature space $\mathbb{K}^{n}$ to the point $(\boldsymbol{u}, \mu(r))$ given in polar coordinates of the tangent space $T_{O} \mathbb{K}^{n}$. The projector function $\mu$ is given in the table

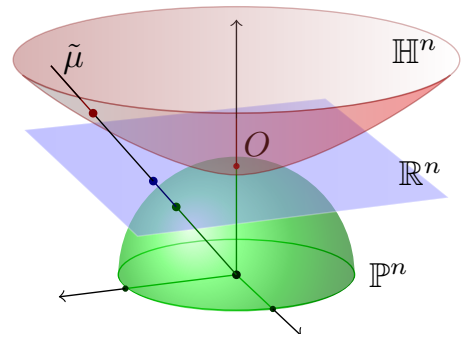

\begin{tabular}{|c||c|c|c|}
\hline $\mathbb{K}^{n}$ & $\kappa$ & $\nu$ & $\mu$ \\
\hline \hline $\mathbb{H}^{n}$ (hyperbolic) & -1 & $\sinh r$ & $\tanh r$ \\
\hline $\mathbb{R}^{n}$ (Euclidean) & 0 & $r$ & $r$ \\
\hline $\mathbb{S}^{n}$ or $\mathbb{P}^{n}$ (elliptic) & +1 & $\sin r$ & $\tan r$ \\
\hline
\end{tabular}

where $\kappa$ is the curvature, $\nu$ is the so-called size function giving the isometry factor between the geodesic sphere of radius $r$ and the Euclidean sphere of radius $\nu(r)$ (see $[7])$.

Let $A, B$ be different points in a projective-metric space $(\mathcal{M}, d)$ and let $C \in$ $(A B \cap \mathcal{M}) \backslash\{B\}$. Then the real number

$$
\langle A, B ; C\rangle_{d}^{\prime}=\left\{\begin{aligned}
\frac{\mu(d(A, C))}{\mu(d(C, B))}, & \text { if } C \in \overline{A B} \\
-\frac{\mu(d(A, C))}{\mu(d(C, B))}, & \text { otherwise }
\end{aligned}\right.
$$

is called the tangential ratio of the triplet $(A, B, C)$, where $\mu$ is the projector function of the hyperbolic, Euclidean or elliptic space according to the type of $(\mathcal{M}, d)$.

Observe that for constant curvature spaces a tangential ratio $\langle A, B ; O\rangle_{d}^{\prime}$ is nothing else but the affine ratio of the projected points $\tilde{\mu}(A), \tilde{\mu}(B)$, and $\tilde{\mu}(O)=O$, i.e. $\langle A, B ; O\rangle_{d}^{\prime}=(\tilde{\mu}(A), \tilde{\mu}(B) ; O)$. This observation is the idea behind the following result. 
Theorem 2.2. In a constant curvature space $\mathbb{K}^{n}$ with projector function $\mu$ we have the following.

(1) Let $O$ be a point in the open triangle $A B C \triangle$. If $X=A O \cap B C, Y=$ $B O \cap C A$, and $Z=C O \cap A B$, then

$$
\begin{aligned}
\langle A, X ; O\rangle_{d}^{\prime}+\langle B, Y ; O\rangle_{d}^{\prime} & +\langle C, Z ; O\rangle_{d}^{\prime}+2 \\
& =\langle A, X ; O\rangle_{d}^{\prime} \cdot\langle B, Y ; O\rangle_{d}^{\prime} \cdot\langle C, Z ; O\rangle_{d}^{\prime}
\end{aligned}
$$

and the sum of any two of the numbers

$$
p=\frac{\mu(d(A, O))}{\langle A, X ; O\rangle_{d}^{\prime}+1}, q=\frac{\mu(d(B, O))}{\langle B, Y ; O\rangle_{d}^{\prime}+1}, \text { and } r=\frac{\mu(d(C, O))}{\langle C, Z ; O\rangle_{d}^{\prime}+1}
$$

is bigger than the third one.

(2) If three segments $\overline{A X}, \overline{B Y}$, and $\overline{C Z}$ has a common point $O$ such that (2.6) is fulfilled, and the sum of any two of the numbers in (2.7) is bigger than the third one, then the segments $\overline{A X}, \overline{B Y}$, and $\overline{C Z}$ can be turned around $O$ so that $X=A O \cap B C, Y=B O \cap C A$, and $Z=C O \cap A B$.

Proof. Consider the tangent space $T_{O} \mathbb{K}^{n}$ at point $O$, and project the triangle into $T_{O} \mathbb{K}^{n}$ by the projector map $\tilde{\mu}$. In the tangent space $T_{O} \mathbb{K}^{n}$ use Theorem 2.1 for the projected triangle and use the easy observation preceding the theorem to get the statements of this theorem.

Observe that (2.4) fails for non-vanishing curvatures, because $\mu$ is not linear in this case. However the equivalent version (2.3) gives

$$
1=\frac{1}{\langle A, X ; O\rangle_{d}^{\prime}+1}+\frac{1}{\langle B, Y ; O\rangle_{d}^{\prime}+1}+\frac{1}{\langle C, Z ; O\rangle_{d}^{\prime}+1} .
$$

We will need the following ellipse characterization. According to [11, p. 64], a point $O$ is called a projective center of the set $\mathcal{S} \subseteq \mathbb{P}^{n}$, if there is a projectivity $\varpi$ such that $\varpi(O)$ is the affine center of $\varpi(\mathcal{S})$.

Lemma 2.3. If the nonempty open convex set $\mathcal{M}$ in the plane is such that any five different points $P, P_{1}, \ldots, P_{4}$ of $\partial \mathcal{M}$, ordered as $P_{1} \prec P_{2} \prec P_{3} \prec P_{4} \prec P_{1}$ for a circuit of $\partial \mathcal{M}$, determine such an ellipse $\mathcal{E}$ that ray $\bar{P} O$ intersects $\mathcal{E}$ in a point of $\partial \mathcal{M}$, where $O=\overline{P_{1} P_{3}} \cap \overline{P_{2} P_{4}}$, then $\partial \mathcal{M}$ is an ellipse.

Proof. Take a point $O \in \mathcal{M}$, and let $\ell_{o}$ and $\ell_{e}$ be any two different straight lines through $O$. Let $\left\{P_{1}, P_{3}\right\}=\ell_{o} \cap \partial \mathcal{M}$ and $\left\{P_{2}, P_{4}\right\}=\ell_{e} \cap \partial \mathcal{M}$. For any point $P \in \partial \mathcal{M}$ let $\mathcal{E}$ be the ellipse that goes through the points $P, P_{1}, \ldots, P_{4}$. Then the condition of the lemma says that ray $\bar{P} O$ intersects $\mathcal{E}$ in a point of $\partial \mathcal{M}$.

Take the projectivity $\varpi$ that maps the points $O, P_{1}, \ldots, P_{4}$ to $(0,0),(-1,1)$, $(1,1),(1,-1)$, and $(-1,-1)$, respectively. Then the ellipse $\varpi(\mathcal{E})$ goes through the points $(-1,1),(1,1),(1,-1)$, and $(-1,-1)$, hence it is symmetric in $(0,0)=\varpi(O)$. 
Further, as projectivities are bijective, ray $\overline{\varpi(P)}(0,0)$ intersects $\varpi(\mathcal{E})$ in a point of $\varpi(\partial \mathcal{M})$. As $\varpi(\mathcal{E})$ is symmetric in $(0,0)$, and $P$ is an arbitrary point of $\partial \mathcal{M}$, it follows that $(0,0)$ is an affine center of $\mathcal{M}$. Thus, $O$ is a projective center of $\mathcal{M}$.

As every point of $\mathcal{M}$ is a projective center of $\mathcal{M}$, it follows by [11, Theorem $3.3(\mathrm{a})]$ that $\partial \mathcal{M}$ is an ellipse.

\section{Euler's RATio-sum formula in Hilbert planes}

Let $\mathcal{M}$ be an open, strictly convex, bounded domain in $\mathbb{R}^{n}$ with boundary $\partial \mathcal{M}$. The function $d: \mathcal{M} \times \mathcal{M} \rightarrow \mathbb{R}$ defined by

$$
d(A, B)= \begin{cases}0, & \text { if } A=B, \\ \frac{1}{2}|\ln (P, Q ; A, B)|, & \text { if } A \neq B, \text { where } \overline{P Q}=\mathcal{M} \cap A B,\end{cases}
$$

is a metric on $\mathcal{M}$ [1, page 297], and is called the Hilbert metric on $\mathcal{M}$. It satisfies the strict triangle inequality, i.e. $d(A, C)+d(C, B)=d(A, B)$ is valid if and only if $C \in \overline{A B}$. A pair $(\mathcal{M}, d)$, where $d$ is the Hilbert metric for its domain $\mathcal{M}$, is called a Hilbert geometry. Notice that Hilbert geometries are projective-metric spaces of hyperbolic type.

Lemma 3.1. Let $A, B$, and $C$ be collinear points in the Hilbert geometry $(\mathcal{M}, d)$, and take an affine coordinate system $A B \rightarrow \mathbb{R}$ such that for the points in $\{P, Q\}=$ $A B \cap \partial \mathcal{M}$ we have $P \mapsto 0, Q \mapsto q$. If $A \mapsto a, B \mapsto b$, and $C \mapsto c$, then the tangential ratio of the triplet $(A, B, C)$ is

$$
\langle A, B ; C\rangle_{d}^{\prime}=(A, B ; C) \frac{(b+c) q-2 b c}{(a+c) q-2 a c} .
$$

Proof. From the first condition of the Lemma we have $0<a<b<q$. Then (3.1) gives

$$
d(A, C)=\frac{1}{2}\left|\ln \left(\frac{c}{a} \frac{q-a}{q-c}\right)\right| \text { and } d(C, B)=\frac{1}{2}\left|\ln \left(\frac{b}{c} \frac{q-c}{q-b}\right)\right| .
$$

As tanh $d=\frac{e^{d}-e^{-d}}{e^{d}+e^{-d}}=\frac{e^{2 d}-1}{e^{2 d}+1}$, these imply

$$
\tanh d(A, C)=\frac{|c-a| q}{(a+c) q-2 a c}, \quad \text { and } \tanh d(C, B)=\frac{|b-c| q}{(b+c) q-2 b c},
$$

hence the formula in the lemma.

Observe that by (3.2) we have

$$
\langle A, B ; C\rangle_{d}^{\prime}=(A, B ; C)\left(\frac{b+c}{a+c}-\frac{2 c^{2}(b-a)}{(a+c) q-2 a c}\right) .
$$

This shows that $\langle A, B ; C\rangle_{d}^{\prime}$ is a strictly monotone function of $q$. It is strictly increasing if $(A, B ; C)>0$, and strictly decreasing if $(A, B ; C)<0$. 
Theorem 3.2. A Hilbert geometry is hyperbolic if and only if it satisfies Euler's ratio-sum formula for every trigon.

Proof. Let $(\mathcal{M}, d)$ be a Hilbert geometry satisfying Euler's ratio-sum formula for every trigon. We prove that it is hyperbolic.

First, we observe that a Hilbert geometry satisfies Euler's ratio-sum formula if and only if its any projective image satisfies Euler's ratio-sum, hence we can assume that $\mathcal{M}$ is bounded. Now, it is sufficient to prove that $\mathcal{M}$ is an ellipse.

By $[2,(16.12)$, p. 91] we have that

a convex body in $\mathbb{R}^{n}(n \geq 3)$ is an ellipsoid if and only if for any

fixed $k \in\{2, \ldots, n-1\}$ every $k$-plane through the origin intersects

it in a $k$-dimensional ellipsoid,

so we need to prove the statement of the theorem only in the plane.

Conducting an indirect proof, assume that $\partial \mathcal{M}$ is not an ellipse, and Euler's ratio-sum formula still holds for every triangle in $\mathcal{M}$.

As $\mathcal{M}$ is not an ellipse, it follows by Lemma 2.3 that there exists such an ellipse $\mathcal{E}$ that $\mathcal{E} \cap \partial \mathcal{M}$ has five different points $P_{1}, \ldots, P_{5}$ such that $\overline{P_{1} P_{3}}$ meets $\overline{P_{2} P_{4}}$ in a point $O$, and ray $\overline{P_{5}} O$ intersects $\mathcal{E}$ and $\partial \mathcal{M}$ in different points $E$ and $M$, respectively. Then, open segments $\overline{P_{1} P_{3}}$ and $\overline{P_{2} P_{4}}$ are straight lines of the Hilbert geometries $(\mathcal{M}, d)$ and $\left(\mathcal{E}, d_{\mathcal{E}}\right)$, while $\overline{P_{5} E}$ is a straight line of $\left(\mathcal{E}, d_{\mathcal{E}}\right)$, and $\overline{P_{5} M}$ is a straight line of $(\mathcal{M}, d)$.

Take a triangle $A B C \triangle$ such that $A \in \overline{O E} \cap \overline{O M}, B \in \overline{O P_{4}}$, and $C \in \overline{O P_{1}}$. Let $X=O A \cap \overline{B C}, Y=O B \cap \overline{C A}$, and $Z=O C \cap \overline{A B}$ (see Figure 3.1).

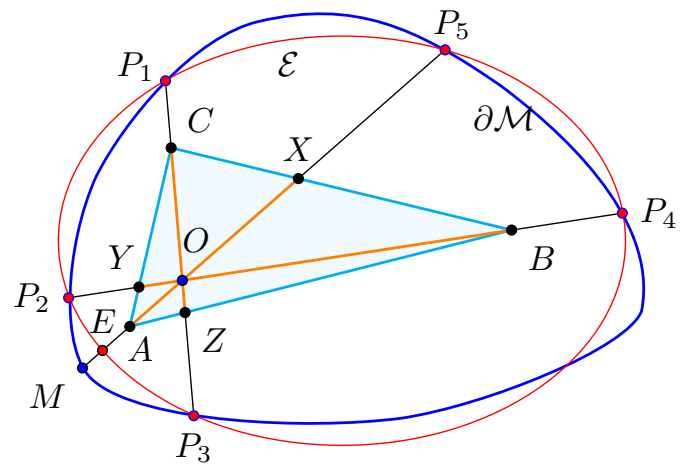

Figure 3.1. Construction of common triangles

Triangle $A B C \triangle$ is in $(\mathcal{M}, d)$ and also in $\left(\mathcal{E}, d_{\mathcal{E}}\right)$, so Euler's tangential ratio-sum formula (2.8) is valid for both, so

$$
\begin{array}{r}
\frac{1}{\langle A, X ; O\rangle_{d}^{\prime}+1}+\frac{1}{\langle B, Y ; O\rangle_{d}^{\prime}+1}+\frac{1}{\langle C, Z ; O\rangle_{d}^{\prime}+1}=1, \\
\frac{1}{\langle A, X ; O\rangle_{d_{\mathcal{E}}}^{\prime}+1}+\frac{1}{\langle B, Y ; O\rangle_{d_{\mathcal{E}}}^{\prime}+1}+\frac{1}{\langle C, Z ; O\rangle_{d_{\mathcal{E}}}^{\prime}+1}=1 .
\end{array}
$$


Since segments $\overline{P_{2} P_{4}}$ and $\overline{P_{1} P_{3}}$ are common straight lines in both geometries, the second and third terms in the left-hand sides of these equations are equal, hence $\langle A, X ; O\rangle_{d}^{\prime}=\langle A, X ; O\rangle_{d_{\mathcal{E}}}^{\prime}$ follows. This contradicts (3.3), hence the proof is complete.

\section{EULER's RATIO-SUM FORMULA IS CHARACTERISTIC}

Lemma 4.1. If a projective-metric space fulfills Euler's tangential ratio-sum formula (2.6) for every triangle, then for any four collinear points $A, B, Z, T$ satisfying $(Z, A ; T, B)=-1$, we have

$$
1=2 \frac{\mu(d(T, Z))}{\mu(d(A, T))+\mu(d(T, Z))}+\frac{\mu(d(T, A))}{\mu(d(B, T))+\mu(d(T, A))} .
$$

Proof. So, we have a projective-metric space $(\mathcal{M}, d)$ satisfying $(2.8)$ with the projector function $\mu$ given appropriately to the type of $(\mathcal{M}, d)$.

If $(\mathcal{M}, d)$ is of elliptic type, then let us cut out a projective line and consider the remaining part with the inherited metric (this is a restriction of $d$ so we denote it with the same letter $d$ ). This way we can consider the triangles in an affine plane independently of the type of $(\mathcal{M}, d)$.

Let us take a segment $\overline{A Z}$, a point $B$ such that $Z \in \overline{A B}$, and a point $C$ out of line $A B$. Let $X \in \overline{B C}$ be such that $Z X \| A C$. Finally, let $O$ be the point of intersection of segments $\overline{A X}$ and $\overline{C Z}$, while $Y=\overline{A C} \cap B O$.

Map triangle $A B C \triangle$ continuously into the degenerate triangle $A A B \triangle$ via the axial affinity with axis $A B$ and moving point $C$ along the segment $\overline{C A}$. That is, $C \rightarrow A, X \rightarrow Z$, and $O$ goes to a point $T$ on $\overline{A B}$.

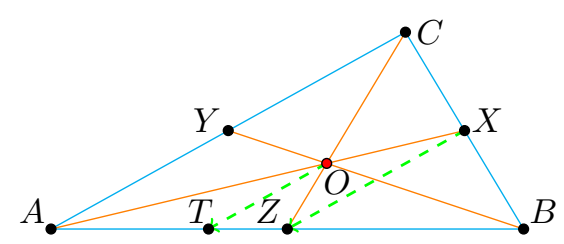

As $d$ and $\mu$ are continuous functions, we obtain

$$
\begin{aligned}
& \frac{\mu(d(O, X))}{\mu(d(A, O))+\mu(d(O, X))} \rightarrow \frac{\mu(d(T, Z))}{\mu(d(A, T))+\mu(d(T, Z))}, \\
& \frac{\mu(d(O, Y))}{\mu(d(B, O))+\mu(d(O, Y))} \rightarrow \frac{\mu(d(T, A))}{\mu(d(B, T))+\mu(d(T, A))}, \text { and } \\
& \frac{\mu(d(O, Z))}{\mu(d(C, O))+\mu(d(O, Z))} \rightarrow \frac{\mu(d(T, Z))}{\mu(d(A, T))+\mu(d(T, Z))} .
\end{aligned}
$$

Substituting these into (2.8) leads to (4.1).

In the other hand, as $Z X \| A C$ and also $T O \| A C$, the intercept theorem gives $(Z, A ; T)=-(Z, A ; B)$, which proves the theorem. 
Theorem 4.2. A projective-metric space of elliptic type fulfills Euler's tangential ratio-sum formula (2.6) for every triangle if and only if it is the elliptic geometry.

Proof. By Lemma (4.1) we have

$$
1=\frac{2 \tan (d(T, Z))}{\tan (d(A, T))+\tan (d(T, Z))}+\frac{\tan (d(T, A))}{\tan (d(B, T))+\tan (d(T, A))}
$$

for every collinear points $A, B, Z, T$ satisfying $(Z, A ; T, B)=-1$.

Let function $P: \mathbb{R} \rightarrow A Z$ be such that $P(s)=Z+s \boldsymbol{v}, A=P(y), B=P(-x)$, and $T=P(t)$. Further, let $\ell: Z A \rightarrow \mathbb{R}$ such that $\ell(s)=\tan (d(P(s), Z))$.

Observe that $(P(x), P(-x) ; Z, P(-\infty))=-1$, so $(4.2)$ gives $\ell(x)=d(P(x), Z)=$ $d(Z, P(-x))=\ell(-x)$, hence $\ell$ is an even function.

Using tangential addition formulas $\tan (d(A, T))=\frac{\ell(y)-\ell(t)}{1+\ell(y) \ell(t)}$ and $\tan (d(B, T))=$ $\frac{\ell(x)+\ell(t)}{1-\ell(x) \ell(t)}$ in $(4.2),(4.2)$ becomes

$$
1=2 \frac{\ell(t)(1+\ell(y) \ell(t))}{\ell(y)\left(1+\ell^{2}(t)\right)}+\frac{(\ell(y)-\ell(t))(1-\ell(x) \ell(t))}{\ell(x) \ell(y)\left(1+\ell^{2}(t)\right)} .
$$

Multiplication by $\ell(x) \ell(y)\left(1+\ell^{2}(t)\right)$ and rearrangement of this result in $\ell(t)=$ $\frac{\ell(x) \ell(y)}{\ell(y)+2 \ell(x)}$. As $(Z, A ; T, B)=-1$, we have $t=\frac{x y}{y+2 x}$, hence

$$
\ell\left(\frac{x y}{y+2 x}\right)=\frac{\ell(x) \ell(y)}{\ell(y)+2 \ell(x)} .
$$

Define the function $f(z)=1 / \ell(1 / z)$ for $z \neq 0$. Then (4.3) mutates to

$$
f\left(\frac{1}{x}+\frac{2}{y}\right)=f\left(\frac{1}{x}\right)+2 f\left(\frac{1}{y}\right) .
$$

Differentiation of this by $x$ immediately gives that $f^{\prime}$ is a constant, and so $f$ is linear. Substituting a linear function into (4.4) clearly shows that the constant vanishes, so we obtain that $\ell(x)=c x$ for some $c>0$ and every $x$. By the definition of $\ell$ this gives $d(P(s), P(0))=\arctan (c s)$ which implies $c=1$, and so the theorem.

Theorem 4.3. A projective-metric space of parabolic type fulfills Euler's tangential ratio-sum formula (2.6) for every triangle if and only if it is a Minkowski geometry.

Proof. By Lemma (4.1) we have

$$
1=2 \frac{d(T, Z)}{d(A, T)+d(T, Z)}+\frac{d(T, A)}{d(B, T)+d(T, A)}
$$

for every collinear points $A, B, Z, T$ satisfying $(Z, A ; T, B)=-1$.

If $B$ goes to any infinity point $B^{*}$ of the affine line $Z A$, then $(Z, A ; T, B)=-1$ implies that $T$ tends to the affine midpoint $T^{*}$ of the affine segment $\overline{A Z}$. This implies $d\left(B^{*}, T^{*}\right)=\infty$, so $1=2 \frac{d\left(T^{*}, Z\right)}{d\left(A, T^{*}\right)+d\left(T^{*}, Z\right)}$, follows from (4.5). This means that $d\left(A, T^{*}\right)=d\left(T^{*}, Z\right)$. 
Thus, the affine and the $d$-metric midpoint of any segment coincide, so, according to Busemann [2, page 94], $d$ is a Minkowski metric.

Theorem 4.4. A projective-metric space of hyperbolic type fulfills Euler's tangential ratio-sum formula (2.6) for every triangle if and only if it is the hyperbolic geometry.

Proof. By Lemma (4.1) we have

$$
1=2 \frac{\tanh (d(T, Z))}{\tanh (d(A, T))+\tanh (d(T, Z))}+\frac{\tanh (d(T, A))}{\tanh (d(B, T))+\tanh (d(T, A))}
$$

for every collinear points $A, B, Z, T$ satisfying $(Z, A ; T, B)=-1$.

Let $B^{*}$ be the $d$-metric infinity of the hyperbolic line $A Z$ on the ray $\bar{A} Z$, and let point $T^{*}$ be such that $\left(Z, A ; T^{*}, B^{*}\right)=-1$. If $B \rightarrow B^{*}$, then $T \rightarrow T^{*}$, and $d(B, T) \rightarrow \infty$, so $\tan (d(B, T)) \rightarrow 1$, hence (4.6) gives

$$
2=\frac{1}{\tanh \left(d\left(T^{*}, Z\right)\right)}-\frac{1}{\tanh \left(d\left(A, T^{*}\right)\right)} .
$$

If $B^{*}$ is the affine infinity on ray $\bar{A} Z$, then $\left(Z, A ; T^{*}, B^{*}\right)=-1$ implies that $T^{*}$ is the affine midpoint of $\overline{A Z}$.

If there exists a whole affine line in $\mathcal{M}$, then both the $d$-metric infinities coincide with the infinities of the affine line $A Z$, hence (4.7) remains valid after $A$ and $Z$ are exchanged. This is a contradiction, so no whole affine line can exist in $\mathcal{M}$.

If $\bar{A} Z$ in $\mathcal{M}$ is a ray, then denote the $d$-metric infinity on the ray $A \bar{Z}$ by $A^{*}$, and let function $P:[0, \infty] \rightarrow \overline{A^{*}} A$ be such that $P(t)=A^{*}+t \boldsymbol{v}$, where $A=P(s)$, $T^{*}=P(s+x)$, hence $Z=P(s+2 x)$. Then (4.7) says that

$$
2=\frac{1}{\tanh (d(P(s+x), P(s+2 x)))}-\frac{1}{\tanh (d(P(s), P(s+x)))}
$$

for every $s \geq 0$ and $x>0$. This implies step-by-step that

$$
\begin{aligned}
2+1=2+\frac{1}{\tanh (d(P(0), P(y)))} & =\frac{1}{\tanh (d(P(y), P(2 y)))}, \\
2+\frac{1}{\tanh (d(P(y), P(2 y)))} & =\frac{1}{\tanh (d(P(2 y), P(3 y)))}, \\
& \vdots \\
2+\frac{1}{\tanh (d(P((k-1) y), P(k y)))} & =\frac{1}{\tanh (d(P(k y), P((k+1) y)))},
\end{aligned}
$$

and the sum of all these equations give

$$
1+2 k=\frac{1}{\tanh (d(P(k y), P((k+1) y)))}
$$


for every $y$. In other form,

$$
d\left(P(z), P\left(\frac{k+1}{k} z\right)\right)=\tanh ^{-1}\left(\frac{1}{1+2 k}\right)=\frac{1}{2} \ln \left(\frac{1+\frac{1}{1+2 k}}{1-\frac{1}{1+2 k}}\right)=\frac{1}{2} \ln \left(\frac{k+1}{k}\right) .
$$

Hence

$\lim _{h \rightarrow 0} \frac{d(P(z), P(z+h))}{h}=\lim _{k \rightarrow \infty} \frac{d\left(P(z), P\left(z+\frac{1}{k} z\right)\right)}{z / k}=\frac{1}{2 z} \lim _{k \rightarrow \infty} \ln \left(\left(1+\frac{1}{k}\right)^{k}\right)=\frac{1}{2 z}$.

Integration of this results in

$$
d(x, y)=\int_{x}^{y} \frac{1}{2 z} d z=\frac{\ln y-\ln x}{2}=\frac{1}{2} \ln \frac{y}{x},
$$

so the metric, on rays, is $d(C, D)=\frac{1}{2} \ln \left(A^{*}, \infty ; C, D\right)$, which is the Hilbert metric.

If $A Z \cap \mathcal{M}=\overline{A^{*} B^{*}}$ is a chord of $\partial \mathcal{M}$, then let function $P:[0,1] \rightarrow \overline{A^{*} B^{*}}$ be such that $P(0)=A^{*}+t\left(B^{*}-A^{*}\right)$. Let $A=P(s), T=P(s+x), Z=P(s+z)$ and $B=P(s+y)$. Then, by the intercept theorem, $(z-x): x=(y-z): y$, i.e. $(x+y) z=2 x y$. If $B \rightarrow B^{*}$, then $y=1-s$, so $(x+1-s) z=2 x(1-s)$. So $(4.7)$ says that

$$
2+\frac{1}{\tanh (d(P(s), P(s+x)))}=\frac{1}{\tanh \left(d\left(P(s+x), P\left(s+\frac{2 x(1-s)}{x+1-s}\right)\right)\right)} .
$$

This implies step-by-step that

$$
\begin{aligned}
2+1=2+\frac{1}{\tanh (d(P(0), P(y)))} & =\frac{1}{\tanh \left(d\left(P(y), P\left(\frac{2 y}{1+y}\right)\right)\right)}, \\
2+\frac{1}{\tanh \left(d\left(P(y), P\left(\frac{2 y}{1+y}\right)\right)\right)} & =\frac{1}{\tanh \left(d\left(P\left(\frac{2 y}{1+y}\right), P\left(\frac{3 y}{1+2 y}\right)\right)\right)}, \\
2+\frac{1}{\left.\tanh \left(d\left(P\left(\frac{2 y}{1+y}\right), P\left(\frac{3 y}{1+2 y}\right)\right)\right)\right)} & =\frac{1}{\tanh \left(d\left(P\left(\frac{3 y}{1+2 y}\right), P\left(\frac{4 y}{1+3 y}\right)\right)\right)}, \\
2+\frac{1}{\left.\tanh \left(d\left(P\left(\frac{k y}{1+(k-1) y}\right), P\left(\frac{(k+1) y}{1+k y}\right)\right)\right)\right)} & =\frac{1}{\tanh \left(d\left(P\left(\frac{(k+1) y}{1+k y}\right), P\left(\frac{(k+2) y}{1+(k+1) y}\right)\right)\right)},
\end{aligned}
$$

and the sum of all these equations give

$$
1+2(k+1)=\frac{1}{\tanh \left(d\left(P\left(\frac{(k+1) y}{1+k y}\right), P\left(\frac{(k+2) y}{1+(k+1) y}\right)\right)\right.}
$$

for every $x$. In other form,

$$
d\left(P(z), P\left(\frac{(k+1) z}{k+z}\right)\right)=\tanh ^{-1}\left(\frac{1}{1+2 k}\right)=\frac{1}{2} \ln \left(\frac{1+\frac{1}{1+2 k}}{1-\frac{1}{1+2 k}}\right)=\frac{1}{2} \ln \left(\frac{k+1}{k}\right) .
$$


Hence

$$
\begin{aligned}
\lim _{h \rightarrow 0} \frac{d(P(z), P(z+h))}{h} & =\lim _{k \rightarrow \infty} \frac{\left.d\left(P(z), P\left(z+\frac{z(1-z)}{k+z}\right)\right)\right)}{\frac{z(1-z)}{k+z}}=\lim _{k \rightarrow \infty} \frac{\frac{1}{2} \ln \left(\frac{k+1}{k}\right)}{\frac{z(1-z)}{k+z}} \\
& =\frac{1}{2 z(1-z)} \lim _{k \rightarrow \infty} \ln \left(\left(1+\frac{1}{k}\right)^{k+z}\right)=\frac{1}{2 z(1-z)} .
\end{aligned}
$$

By integrating this, we get

$$
\begin{aligned}
d(x, y) & =\int_{x}^{y} \frac{1}{2 z(1-z)} d z=\frac{1}{2} \int_{x}^{y} \frac{1}{z}+\frac{1}{1-z} d z \\
& =\frac{(\ln y-\ln x)-(\ln (1-y)-\ln (1-x))}{2}=\frac{1}{2} \ln \frac{y:(1-y)}{x:(1-x)},
\end{aligned}
$$

so, on chords $\overline{A^{*} B^{*}}, d(C, D)=\frac{1}{2} \ln \left(A^{*}, B^{*} ; C, D\right)$, which is the Hilbert metric.

Thus, $(\mathcal{M}, d)$ is a Hilbert geometry, and so Theorem 3.2 finishes the proof.

In sum, the above results prove the following main result of this paper.

Theorem 4.5. A projective-metric space fulfills Euler's tangential ratio-sum formula (2.6) for every triangle if and only if it is a Minkowski geometry, the hyperbolic geometry, or the elliptic geometry.

\section{REFERENCES}

[1] H. Busemann and P. J. Kelly, Projective Geometries and Projective Metrics, Academic Press, New York, 1953. $\langle 2,5\rangle$

[2] H. Busemann, The Geometry of Geodesics, Academic Press, New York, 1955. $\langle 2,6,9\rangle$

[3] L. Euler, Geometrica et sphaerica quaedam, Memoires de lAcademie des Sciences de Saint-Petersbourg, 5 (1815), 96-114; Opera Omnia Series 1, vol. XXVI, 344-358; Original: http://eulerarchive.maa.org/docs/originals/E749.pdf; English translation: http: //eulerarchive.maa.org/Estudies/E749t.pdf. $\langle 1,2\rangle$

[4] B. Grünbaum and M. S. Klamkin, Euler's Ratio-Sum Theorem and Generalizations, Mathematics Magazine, 79:2(Apr) (2006), 122-130; http://www.jstor.org/stable/27642919. $\langle 1\rangle$

[5] B. Grünbaum, Cyclic ratio sums and products, Crux Mathematicorum, 24:1 (1998), 20-25; https://cms .math.ca/crux/v24/n1/page20-25.pdf. $\langle 1\rangle$

[6] J. Kozma and Á. Kurusa, Hyperbolic is the only Hilbert geometry having circumcenter or orthocenter generally, Beiträge zur Algebra und Geometrie, 57:1 (2016), 243-258; DOI: $10.1007 / \mathrm{s} 13366-014-0233-3$.

[7] Á. Kurusa, Support Theorems for Totally Geodesic Radon Transforms on Constant Curvature Spaces, Proc. Amer. Math. Soc., 122:2(1994), 429-435; DOI: 10.2307/2161033. $\langle 3\rangle$

[8] Á. Kurusa and J. Kozma, Euler's ratio-sum theorem revisited, (submitted). $\langle 2\rangle$

[9] A. J. Lexell, Solutio problematis geometrici ex doctrina sphaericorum, Acta academiae scientarum imperialis Petropolitinae, 5:1 (1784), 112-126; http://www.17centurymaths.com/ contents/euler/lexellone.pdf. $\langle 1,2\rangle$

[10] A. Papadopoulos and W. Su, On hyperbolic analogues of some classical theorems in spherical geometry, arXiv,(2015), 1409.4742. $\langle 1,2\rangle$

[11] L. Montejano and E. Morales, Characterization of ellipsoids and polarity in convex sets, Mathematika, 50 (2003), 63-72; DOI: 10.1112/S0025579300014790. $\langle 4,5\rangle$ 
[12] G. C. Shephard, Euler's Triangle Theorem, Crux Mathematicorum, 25:3 (1999), 148-153; https://cms.math.ca/crux/v25/n3/page148-153.pdf. $\langle 1\rangle$

[13] C. E. SAndifer, 19th century Triangle Geometry (May 2006), How Euler did it, Math. Ass. Amer., 2007, 19-27; http://eulerarchive.maa.org/hedi/HEDI-2006-05.pdf. $\langle 1\rangle$

[14] Z. I. Szabó, Hilbert's fourth problem. I, Adv. in Math., 59:3 (1986), 185-301; DOI: 10.1016/0001-8708(86)90056-3. $\langle 2\rangle$

Á. Kurusa, Bolyai Institute, University of Szeged, Aradi vértanúk tere 1, 6725 Szeged, Hungary; and Alfréd Rényi Institute of Mathematics, Hungarian Academy of Sciences, Reáltanoda u. 13-15, H-1053 Budapest, Hungary; E-mail: kurusa@math.u-szeged.hu.

J. Kozma, Bolyai Institute, University of Szeged, Aradi vértanúk tere 1, 6725 Szeged (Hungary); E-mail: kozma@math.u-szeged.hu . 